\title{
Deformable Modeling for Characterizing Biomedical Shape Changes
}

\author{
Matthieu Ferrant ${ }^{2}$, Benoit Macq $^{2}$, Arya Nabavi ${ }^{1}$, and Simon K. Warfield ${ }^{1}$ \\ 1 Surgical Planning Laboratory, Brigham and Women's Hospital \\ Harvard Medical School, Boston, USA \\ \{warfield, arya\}@bwh.harvard.edu \\ 2 Telecommunications Laboratory, Université catholique de Louvain, Belgium \\ $\{$ ferrant, macq\}@tele.ucl.ac.be
}

\begin{abstract}
We present a new algorithm for modeling and characterizing shape changes in 3D image sequences of biomedical structures. Our algorithm tracks the shape changes of the objects depicted in the image sequence using an active surface algorithm. To characterize the deformations of the surrounding and inner volume of the object's surfaces, we use a physics-based model of the objects the image represents. In the applications we are presenting, our physics-based model is linear elasticity and we solve the corresponding equilibrium equations using the Finite Element (FE) method. To generate a FE mesh from the initial 3D image, we have developed a new multiresolution tetrahedral mesh generation algorithm specifically suited for labeled image volumes. The shape changes of the surfaces of the objects are used as boundary conditions to our physics-based FE model and allow us to infer a volumetric deformation field from the surface deformations. Physics-based measures such as stress tensor maps can then be derived from our model for characterizing the shape changes of the objects in the image sequence. Experiments on synthetic images as well as on medical data show the performances of the algorithm.
\end{abstract}

Keywords: Deformable models, Active surface models, Finite elements, Tetrahedral mesh generation

\section{Introduction}

Today, there is a growing need for physics-based image analysis of deformations in image sequences (e.g. real-time MRI of the heart, image sequences showing brain deformation during neurosurgery, etc.). The subject has recently lead to considerable interest in the medical image analysis community $[1,2,3,4,5,6,7,8]$.

Medical image analysis has in the past relied heavily upon qualitative description. Today, modern applications can be enabled by providing to the clinician quantitative data derived from these images. For example, rather than simply observing erratic heart beat with real-time MRI, clinicians want to measure ejection fraction and estimate stress in the heart muscle quantitatively. 
Shape- and surface-based image analysis is being increasingly used in the bio-medical image analysis community, e.g., for pathological analysis [9] and for tracking deformations [10]. Shape-based models are also being used for image segmentation $[11,12,13]$ to constrain active surface models $[1,14]$. Such active surface models do not allow any physical interpretation of the deformation the surfaces undergo. Also, no volumetric deformation field is available.

In an attempt to overcome these problems, several authors have proposed to use a physics-based model to infer a volumetric deformation field (e.g. $[15,16])$ from surface-based deformations. But the used parameters were determined heuristically, and one could therefore not exploit the information generated by the model to extract biomechanical properties. Other authors have also proposed to use physical deformation models to constrain a volumetric deformation field computed from image data using elastic [17] or even viscous fluid deformation models $[18,19]$. But in these applications, the models did not account for actual material characteristics, because the matching is done minimizing an energy measure that consists of a weighted sum of an image similarity term and a relaxation term representing the potential energy of a physical body (e.g., elastic). Therefore, the actual physics of the phenomenon cannot be properly captured by these models. In order to capture a physics-based deformation field, one needs to use biomechanical models and image-derived forces for deforming them.

In the context of brain shift analysis, there recently has been a significant amount of work directed towards simulation [7] using models driven by physicsbased forces such as gravity. Skrinjar et al. [4] have proposed a model consisting of mass nodes interconnected by Kelvin models to simulate the behavior of brain tissue under gravity, with boundary conditions to model the interaction of the brain with the skull. Miga et al. [3] proposed a Finite Element (FE) model based on consolidation theory where the brain is modeled as an elastic body with an interstitial fluid. They also use gravity induced forces, as well as experimentally determined boundary conditions.

Even though these models are very promising, it remains difficult to accurately estimate all the forces and boundary conditions that interact with the model.

The cardiac image analysis community has been using physics-based models - mainly FE models - they deform with image-derived forces. These models then provide quantitative, and physically interpretable 3D deformation estimates from image data. Papademetris et al. [20] derive the forces they apply to the FE model from Ultrasound (US) using deformable contours they match from one image to the next one using a shape-tracking algorithm. Metaxas et al. [1] derive their forces from MRI-SPAMM data for doing motion analysis of the left or right ventricle [21,22].

In the context of deformable brain registration, Hagemann et al. [5] use a biomechanical model for registering brain images, but they enforce correspondances between landmark contours manually. Moreover, the basic elements of their FE model are pixels, which causes the computations to be very slow. Kyriacou et al. [23] study the effect of tumor growth in brain images for doing atlas 
registration. They use a $\mathrm{FE}$ model and apply concentric forces to the tumor boundary to shrink it. In these two studies, the experiments were performed in $2 \mathrm{D}$, thereby limiting the clinical utility and the possibility to efficiently assess the accuracy of the methods.

We propose to merge the prior physical knowledge physicians have about the object that is being imaged with the information that can be extracted from the image sequence to obtain quantitative measurements. We extract shape information of the objects in the image sequence using an active surface model, and characterize the changes the objects undergo using a physics-based model.

The idea is similar to that used for cardiac analysis; we track boundary surfaces in the image sequence, and we use the boundary motion as input for a FE model. The boundary motion is used as a boundary condition for the FE model to infer a volumetric deformation field, as proposed in [5].

The main contribution of this paper is that instead of using a generic FE model that is fitted to the image data as it is done for cardiac image analysis [1], or using the pixels (or voxels) as basic elements of the FE model, we propose an algorithm for generating patient-specific tetrahedral FE models from the initial 3D image in the sequence, with locally adaptable resolution, and integrated boundary surfaces. Also, this enables us to perform computations in 3D, without manual interaction, and moreover, on a limited number of elements, with equivalent precision, and in a reasonable amount of time on a common workstation, thanks to an efficient implementation of the FE deformation algorithm.

\section{Description of the Algorithm}

There are two important points for doing physics-based modeling of the deformation in $3 \mathrm{D}$ image sequences. One first needs to have a prior bio-mechanical model of the object represented by the image, i.e., the constitutive equations of the bodies (elastic, fluid, viscous fluid, etc.) represented in the image. On the other hand, one also needs a way of applying forces and boundary conditions to the model using the image information.

In this work, we have chosen to model image structures as elastic bodies. More elaborate models can of course very easily be integrated into our algorithm. Thus, we assume that the objects that are being imaged have an elastic behavior during deformation. The deformations will be tracked using the boundary information of the objects in the image sequence. The boundary surfaces are deformed towards the boundaries of the next 3D image in the sequence using an active surface algorithm. The deformation field of the boundary surfaces is then used as a boundary condition for our bio-mechanical model, that will be used to infer the deformation field throughout the entire volume.

This will provide us with physically realistic and interpretable information (such as stress tensors, compression measures, etc.) of the imaged objects during the whole sequence. 


\section{Mathematical Formulation}

Assuming a linear elastic continuum with no initial stresses or strains, the potential energy of an elastic body submitted to externally applied forces can be expressed as $[24]^{1}$ :

$$
E=\frac{1}{2} \int_{\Omega} \boldsymbol{\sigma}^{T} \boldsymbol{\epsilon} d \Omega+\int_{\Omega} \mathbf{F u} d \Omega
$$

where $\mathbf{u}=\mathbf{u}(x, y, z)$ is the displacement vector, $\mathbf{F}=\mathbf{F}(x, y, z)$ the vector representing the forces applied to the elastic body (forces per unit volume, surface forces or forces concentrated at the nodes), and $\Omega$ the body on which one is working. $\epsilon$ is the strain vector, defined as

$$
\boldsymbol{\epsilon}=\left(\frac{\partial \mathbf{u}}{\partial x}, \frac{\partial \mathbf{u}}{\partial y}, \frac{\partial \mathbf{u}}{\partial z}, \frac{\partial \mathbf{u}}{\partial x}+\frac{\partial \mathbf{u}}{\partial y}, \frac{\partial \mathbf{u}}{\partial y}+\frac{\partial \mathbf{u}}{\partial z}, \frac{\partial \mathbf{u}}{\partial x}+\frac{\partial \mathbf{u}}{\partial z}\right)^{T}=\mathbf{L} \mathbf{u}
$$

and $\boldsymbol{\sigma}$ the stress vector, linked to the strain vector by the constitutive equations of the material. In the case of linear elasticity, with no initial stresses or strains, this relation is described as

$$
\boldsymbol{\sigma}=\left(\sigma_{x}, \sigma_{y}, \sigma_{z}, \tau_{x y}, \tau_{y z}, \tau_{x z}\right)^{T}=\mathbf{D} \boldsymbol{\epsilon}
$$

where $\mathbf{D}$ is the elasticity matrix characterizing the properties of the material [24].

This equation is valid whether one is working with a surface or a volume. We model our active surfaces, which represent the boundaries of the objects in the image, as elastic membranes, and the surrounding and inner volumes as 3D volumetric elastic bodies.

Within a finite element discretization framework, an elastic body is approximated as an assembly of discrete finite elements interconnected at nodal points on the element boundaries. This means that the volumes to be modeled need to be meshed, i.e. divided into elements. Our meshing algorithm will be described in the next section.

The continuous displacement field $\mathbf{u}$ within each element is a function of the displacement at the element's nodal points $\mathbf{u}_{i}^{e l}$ weighted by its shape functions $N_{i}^{e l}=N_{i}^{e l}(x, y, z)(4)$.

$$
\mathbf{u}=\sum_{i=1}^{N_{\text {nodes }}} N_{i}^{e l} \mathbf{u}_{i}^{e l}
$$

The elements we use are tetrahedra $\left(N_{\text {nodes }}=4\right)$ for the volumes and triangles for the membranes $\left(N_{\text {nodes }}=3\right)$, with linear interpolation of the displacement field. Hence, the shape function of node $i$ of tetrahedron $e l$ is defined as:

$$
N_{i}^{e l}(\mathbf{x})=K\left(a_{i}^{e l}+b_{i}^{e l} x+c_{i}^{e l} y+d_{i}^{e l} z\right)
$$

\footnotetext{
${ }^{1}$ Superscript $\mathrm{T}$ designs the transpose of a vector or a matrix
} 
where $K=\frac{1}{6 V^{e t}}$ for a tetrahedron, and $K=\frac{1}{2 S^{e t}}$ for a triangle. The computation of $V^{e l}, S^{e l}$ (volume, surface of $e l$ ) and the other constants is detailed in [24].

For every node $i$ of each element $e l$, we define the matrix $\mathbf{B}_{i}^{e l}=\mathbf{L}_{i} N_{i}^{e l}(\mathbf{x})$. The function to be minimized at every node $i$ of each element $e l$ can thus be expressed as :

$$
E\left(\mathbf{u}_{i}^{e l}\right)=\int_{\Omega} \sum_{j=1}^{N_{\text {nodes }}} \mathbf{u}_{i}^{e l^{T}} \mathbf{B}_{i}^{e l^{T}} \mathbf{D B}_{j}^{e l} \mathbf{u}_{j}^{e l}+\mathbf{F}(\mathbf{x}) N_{i}^{e l}(\mathbf{x}) \mathbf{u}_{i}^{e l} d \Omega
$$

We seek the minimum of this function by solving for $\frac{d E\left(u_{i}^{e l}\right)}{d u_{i}^{e l}}=0$. Equation (6) then becomes :

$$
\int_{\Omega} \sum_{j=1}^{N_{\text {nodes }}} \mathbf{B}_{i}^{e l}{ }^{T} \mathbf{D B}_{j}^{e l} \mathbf{u}_{j}^{e l} d \Omega=-\int_{\Omega} \sum_{j=1}^{N_{\text {nodes }}} \mathbf{F}(\mathbf{x}) N_{i}^{e l}(\mathbf{x}) d \Omega
$$

This last expression can be written as a matrix system for each finite element:

$$
\mathbf{K}^{e l} \mathbf{u}^{e l}=-\mathbf{F}^{e l}
$$

Matrices $\mathbf{K}^{e l}$ and vector $\mathbf{F}^{e l}$ are defined as follows: $\mathbf{K}_{i, j}^{e l}=\int_{\Omega} \mathbf{B}_{i}^{e l}{ }^{T} \mathbf{D B}_{j}^{e l} d \Omega$, $\mathbf{F}_{j}^{e l}=\int_{\Omega} \mathbf{F} N_{i}^{e l} d \Omega$; where every element $i, j$ refers to pairs of nodes of the element $e l$ ( $i$ and $j$ range from 1 to 4 for a tetrahedron -1 to 3 for a triangle). $\mathbf{K}_{i, j}^{e l}$ is a 3 by 3 matrix, and $\mathbf{F}_{j}^{e l}$ is a 3 by 1 vector. The 12 by 12 (9 by 9 for a triangle) matrix $\mathbf{K}^{e l}$, and the vector $\mathbf{F}^{e l}$ are computed for each element and are then assembled in a global system $\mathbf{K u}=-\mathbf{F}$, the solution of which will provide us with the deformation field corresponding to the global minimum of the total energy.

We now have constitutive equations that model surfaces as elastic membranes and volumes as elastic bodies.

\subsection{Finite Element Mesh Generation}

In [5], Hagemann et al. propose to use the pixels of the image as basic elements of his FE mesh. This approach does not take advantage of the intrinsic formulation of FE modeling, which assumes that the mechanical properties are constant over the element, suggesting that one can use elements covering several image pixels. Also, when performing computations in $3 \mathrm{D}$, which is eventually what is needed for medical applications, the amount of degrees of freedom will be far too large (for a typical 256x256x60 MR image, this means about 12 million degrees of freedom at worst case !) to perform efficient computations in a reasonable time, even on high performance computing equipment.

Most available meshing software packages do not allow meshing of multiple objects (e.g., [25,26]), and are usually designed for regular and convex objects, which is often not the case for anatomical structures. Therefore, we have implemented a tetrahedral mesh generator specifically suited for labeled 3D medical 
images. The mesher can be seen as the volumetric counterpart of a marching tetrahedra surface generation algorithm, the only difference being that the initial tetrahedralization we use can have an adaptive resolution with sizes of tetrahedra depending on the underlying image content.

The labeled 3D image from which the mesh needs to be computed is first divided into cubes of a given size, which are further divided into 5 tetrahedra with an alternating pattern so as to avoid diagonal crossings on the shared quadrilateral faces of neighboring cubes. The initial cube size determines the size of the largest tetrahedra the mesh will contain. Each tetrahedron is checked for subdivision according to the underlying image content. In our case, we decided to only subdivide tetrahedra that lie across boundaries of given objects, so as to have a detailed description of their boundaries. The edges of those tetrahedra to be subdivided are labeled for subdivision, and a new vertex is inserted at their middle point. This process is executed iteratively until the smallest edges have reached a specified minimum size.

At each iteration, the mesh is re-tetrahedrized given the required edge subdivisions for each tetrahedron. The main problem is to re-mesh tetrahedra that lie next to tetrahedra that are being split. For those tetrahedra, only some edges have been split. The mesh is therefore re-tetrahedrized using a case table with the $2^{6}=64$ possible edge splitting configurations. There are 10 basic configurations, the others are symmetrical to those presented on Figure 1 (the gray coloring and the different node labelings are represented only to facilitate visualization of the tetrahedra's subdivisions). From upper left to lower right, Figure 1 successively presents the tetrahedrization if one edge is split, if two edges are split (2 possible configurations), if three edges are split (3 possible configurations), if 4 edges are split (3 possible configurations), if 5 edges are split and finally if all edges of the tetrahedron are split.


2 prisms

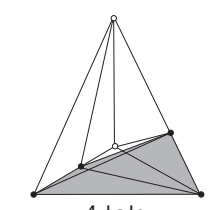

4 tets

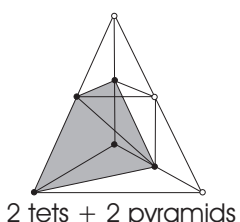

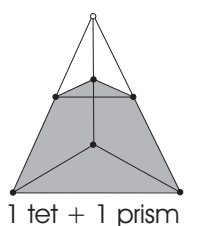

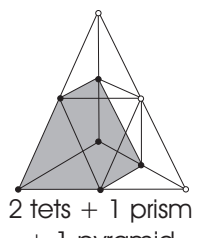

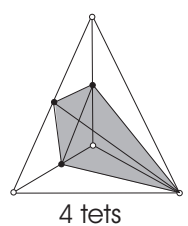

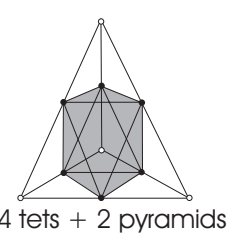

Fig. 1. Different subdivisions of a tetrahedron given edge splittings 
The resulting mesh contains tetrahedra, but also pyramids and prisms, which need to be further tetrahedrized. The main problem is to ensure consistency ${ }^{2}$ between the diagonals of quadrilateral faces shared by 2 elements (pyramids or prisms). We split the quadrilateral faces along the shortest diagonal so as to have better shaped tetrahedra. The subdivision of a pyramid into two tetrahedra is straightforward given the diagonal of the quadrilateral face. For a prism, there are eight possible configurations for tetrahedralization given the diagonal configuration. Figure 2 presents the different possible tetrahedralizations of a prism given the diagonal's configuration. If no straight tetrahedralization is possible (cases 1 and 8), a vertex is inserted in the middle of the prism which is then divided into 8 tetrahedra.

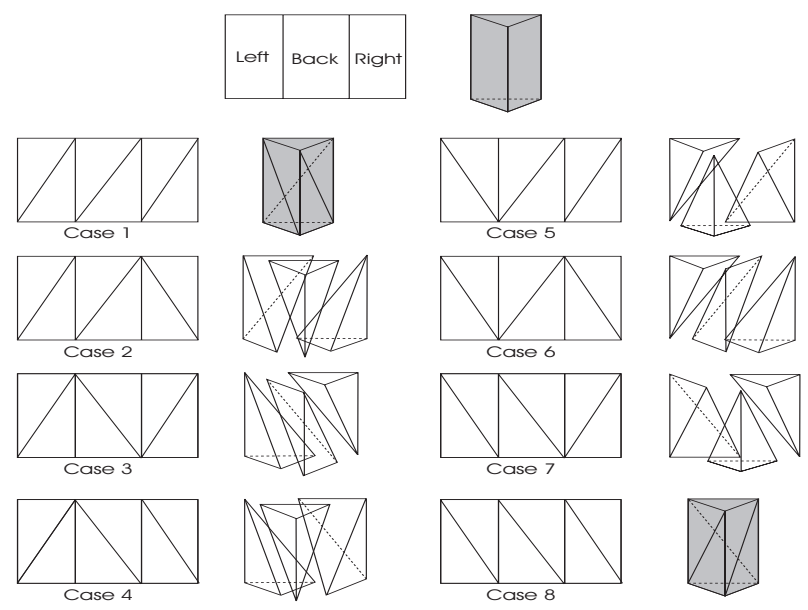

Fig. 2. Different subdivisions of a prism given the quadrilateral faces' diagonals

Finally, we apply a marching tetrahedra-like approach to generate the actual tetrahedral mesh with accurately represented boundary surfaces. For each tetrahedron, the image labels at its nodes are checked. A case table draws the elements to be added to the mesh. If all 4 nodes have non-object labels, no tetrahedron is added to the mesh. If all nodes have an object label, the tetrahedron is added to the mesh as is. If the tetrahedron lies across two objects (i.e. all nodes do not have the same label), the subdivision of the original tetrahedron is looked up in the case table.

Figure 3 shows the 5 basic cases. There are actually 16 cases, but the remaining cases are symmetric to cases 2,3 , and 4 . The resulting prisms are divided into tetrahedra using the same approach as presented above.

The resulting mesh structure is built such that for images containing multiple objects, a fully connected and consistent tetrahedral mesh is obtained for every cell, whith a given label corresponding to the object the cell belongs to.

\footnotetext{
${ }^{2}$ A consistent tetrahedral mesh is built such that every (non-boundary) triangular face is shared by exactly 2 tetrahedra.
} 

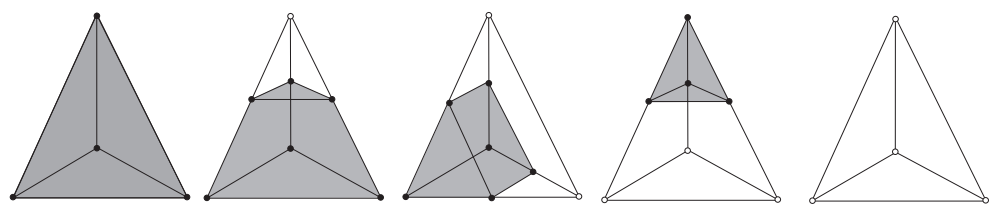

Fig. 3. Different tetrahedral cases depicted from left to right. Case 1: all nodes belong to structure; case 2: 3 nodes belong to structure; case 3: 2 nodes belong to structure; case 4: 1 node belongs to structure; case 5: no nodes belong to structure

Therefore, different biomechanical properties and parameters can easily be assigned to the different cells or objects composing the mesh. Boundary surfaces of objects represented in the mesh can be extracted from the mesh as triangulated surfaces, which is very convenient for running an active surface algorithm.

\subsection{Active Surface Algorithm}

The active surface algorithm deforms the boundary surface of an object in one volumetric scan of the sequence towards the boundary of the same object in the next scan of the sequence. This is done iteratively by applying image-derived forces $\mathbf{F}^{\mathbf{u}^{t}}$ (forces computed using the surface's nodal positions $\mathbf{u}$ at iteration $t$ ) to the elastic membrane. The temporal variation of the surface can be discretized using finite differences, provided the time step $\tau$ is small enough. This yields the following semi-implicit iterative equation ${ }^{3}$ :

$$
\frac{\mathbf{u}^{t}-\mathbf{u}^{t-1}}{\tau}+\mathbf{K} \mathbf{u}^{t}=-\mathbf{F}^{\mathbf{u}^{t-1}}
$$

which can be rewritten as :

$$
\left(\mathbf{I}+\tau \mathbf{K} \mathbf{u}^{t}\right)=\mathbf{u}^{t-1}-\tau \mathbf{F}^{\mathbf{u}^{t-1}}
$$

The external forces driving the elastic membrane toward the edges of the structure in the image are integrated over each element of the mesh and distributed over the nodes belonging to the element using its shape functions (see Eqn. 4). Classically, the image force $\mathbf{F}$ is computed as a decreasing function of the gradient so as to be minimized at the edges of the image [14,27]. A potential weakness of active surface methods is that for correct convergence, the surfaces need to be initialized very close to the edges of the object to be segmented. In [14], Cohen et al. proposed to use inflation or deflation forces (so-called balloon forces) to circumvent that problem. To increase the robustness and the convergence rate of the surface deformation, we compute our forces as a gradient descent on a distance map of the edges in the target image. The distance map is computed very efficiently using our fast distance transformation algorithm [28]. To prevent the surface from sticking on a wrong edge, or to prevent two sides of a thin

\footnotetext{
${ }^{3}$ Superscript $t$ refers to the current iteration.
} 
surface from sticking together on the same edge, we have included the expected gradient sign of the structure to be segmented in the force expression. Also, we have signed the distance map for improved convergence. More details about our active surface algorithm can be found in [29].

\subsection{Inferring Volumetric Deformations from Surface Deformations}

The deformation field obtained for the boundary surfaces is then used in conjunction with the volumetric model to infer the deformation field inside and outside the boundary surfaces.

The idea is to apply forces to the boundary surfaces that will produce the same displacement field at the boundary surfaces that was obtained with the active surface algorithm. The volumetric biomechanical model will then compute the deformation of the surrounding nodes in the mesh.

Let $\widetilde{\mathbf{u}}$ be the vector representing the displacement of the boundary nodes to be imposed. Hence, the equilibrium equation of the elastic body (Eqn. 1) needs to be rewritten with the following external forces to impose these displacements to the volume :

$$
\mathbf{F}=\mathbf{K} \widetilde{\mathbf{u}}
$$

The solution of the global equilibrium system (see Eqn. 8) will provide us with the displacement at all the nodes in the volumetric mesh with the imposed displacements at the nodes of the boundary surfaces delimiting the objects represented in the mesh. This volumetric displacement field is then interpolated back onto the image grid using the shape functions of every element of the mesh (see Eqn. 4).

Biomechanical parameters such as the stress tensors can then be derived from the displacements at the nodes using the stress-strain relationship (Eqn. 3) :

$$
\boldsymbol{\sigma}_{i}=\sum_{T e t \mid i \in T e t} \mathbf{D} \boldsymbol{\epsilon}_{i}=\sum_{T e t \mid i \in T e t} \mathbf{D L}_{i} N_{i}^{T e t} \mathbf{u}_{i}
$$

\section{Experiments}

\subsection{Synthetic Image Sequence}

We have tested the algorithm on a sequence of two 3D images of an elastic sphere being squeezed in a given direction. The object is surrounded by another elastic object.

The original active surface extracted from the volumetric tetrahedral mesh is shown in Figure 4b. Note that for this experiment, the initial tetrahedralization from which the mesh was computed was not multi-resolution, it had constant tetrahedral sizes. When running the active surface algorithm, the surface readily converges to the boundary of the ellipsoid in the target image.

Figure 5 shows $3 \mathrm{D}$ views of the mesh associated to the initial image and of the mesh after deformation, while Figure 6 shows cuts through the original 

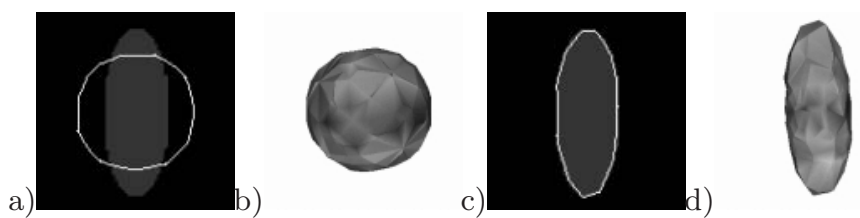

Fig. 4. a) Slice 30 of the target image with a cut through the initial surface of the object overlayed. b) 3D surface rendering of the initial surface. c) The same slice with a cut through the deformed surface. d) 3D surface rendering of the deformed surface

(a) and deformed mesh (b) and the deformation field (c) interpolated back onto the image grid (downsampled for clarity) overlayed on a cut through the target volume. One can very well observe the physical squeezing of the sphere onto the ellipsoid, also deforming the surrounding elastic medium.
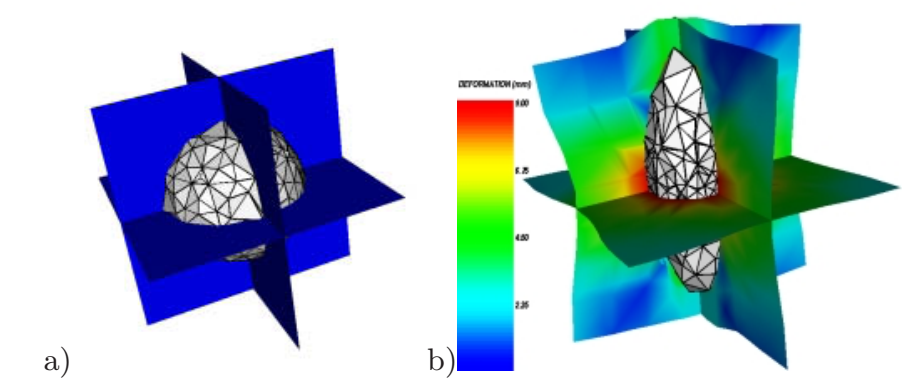

Fig. 5. a) Orthogonal cuts through the initial volumetric mesh with the sphere extracted and b) the same with deformed mesh

\subsection{Brain Shift Analysis}

In this experiment, we wanted to characterize the deformation the brain undergoes during neurosurgery after craniotomy. Two 3D MR volumetric scans were taken before and after craniotomy and partial tumor resection, and a significant shift could be observed. Figure 7 shows cuts through a sample tetrahedral mesh of the brain overlayed on the corresponding initial image.

The active surface is extracted from the intraoperative scan at start of surgery, before opening the dura mater (see Figure 8a), and deformed towards the brain in a later intraoperative image (see Figure 8b). Figure $8 \mathrm{c}$ shows the 3D surface deformation field the brain has undergone. One can observe that the deformation of the cortical surface is happening in the direction of gravity and is mainly located where the dura was removed. Part of the shift (especially on the left of the picture) is also due to the tumor resection that was done between the two scans.

The deformation field obtained with the active surface algorithm is then used as a boundary condition for our biomechanical FE model, and allows us to infer 
a)
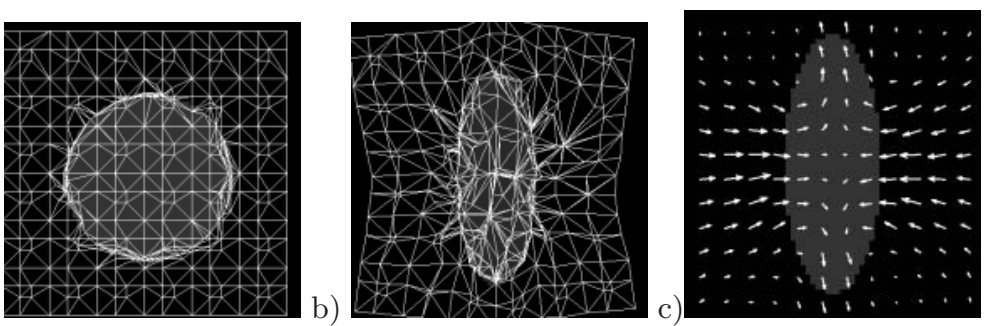

Fig. 6. a) Axial cut through original mesh overlayed on slice of original image and b) the same with deformed mesh on target image. c) The deformation field overlayed on slice of target image
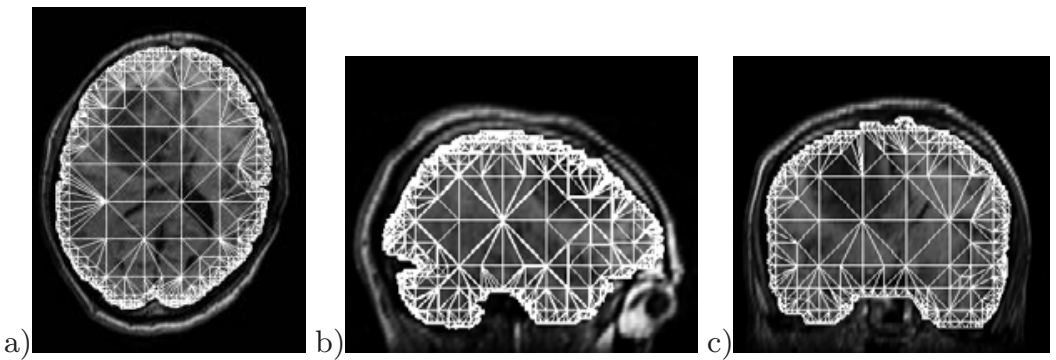

Fig. 7. Axial (a), sagittal (b), and coronal (c) cuts through tetrahedral mesh of the brain overlayed on corresponding cuts through preoperative image

a volumetric deformation field. Elasticity parameters were chosen according to in-vivo studies carried out by Miga et al. [3]. Figure 9a shows the obtained deformation field overlayed on a slice of the initial scan, and Figure 9b shows the same slice of the initial scan deformed with the obtained deformation field. Figure 9 also presents the same slice of the target scan and the magnitude of the difference with the initial scan showing the closeness of the alignment of the brain. The gray-level mean square difference between the target scan and the deformed original scan on the image regions covered by the mesh went down from 181 to 88 . The remaining difference is due to the fact that the model we used did not incorporate the ventricular thinning and the tumor resection that occurred between both scans.

\section{Conclusions}

We have presented a new algorithm for tracking and characterizing shape changes in $3 \mathrm{D}$ image sequences of physics-based objects. The algorithm incorporates a biomechanical model of the deforming objects and uses image-based information to drive the deformation of our model through an active surface algorithm. One of the main contributions of this paper is an improved algorithm for generating multi-resolution patient-specific FE meshes from labeled 3D images. 



Fig. 8. Axial cut through active surface a) initial, and b) deformed, overlayed on corresponding slice of intraoperative MR image. c) 3D surface rendering of active surface with colorcoded intensity of the deformation field


Fig. 9. a) Volumetric deformation field overlayed on initial intraoperative image slice. b) The same slice of initial image deformed using deformation field. c) Same slice of target image. d) The difference between target and deformed images with same slice

The algorithm is a promising tool for the analysis of 3D medical image sequences. It will provide physicians with a tool for measurement and physical interpretation of deformation in 3D image sequences, and can thus be of great aid in in the interpretation and diagnosis of these images.

\section{References}

1. D. M. Metaxas. Physics-Based Deformable Models: Applications to Computer Vision, Graphics and Medical Imaging. Kluwer Academic Publishers, 1997. 235, 236, 237

2. D. L. G. Hill, C. R. Maurer, A. J. Martin, S. Sabanathan, W. A. Hall, D. J. Hawkes, D. Rueckert, and C. L. Truwit. Assessment of intraoperative brain deformation using interventional $\mathrm{mr}$ imaging. In Berlin Springer-Verlag, editor, MICCAI '99, pages $910-919,1999.235$

3. M. I. Miga, K. D. Paulsen, P. J. Hoopes, F. E. Kennedy, A. Hartov, and D. W. Roberts. In vivo quantification of a homogeneous brain deformation model for updating preoperative images during surgery. IEEE Transactions on Medical Imaging, 47(2):266-273, February 2000. 235, 236, 245

4. O. M. Skrinjar and J. S. Duncan. Real time 3d brain shift compensation. In IPMI '99, 1999. 235, 236 
5. A. Hagemann, Rohr K., H. S. Stiel, U. Spetzger, and Gilsbach J. M. Biomechanical Modeling of the Human Head for Physically Based, Non-Rigid Image Registration. IEEE Transactions on Medical Imaging, 18(10):875-884, October 1999. 235, 236, 237, 239

6. Paul M. Thompson, Jay N. Giedd, Roger P. Woods, David MacDonald, Alan C. Evans, and Arthur W. Toga. Growth patterns in the developing brain detected by using continuum mechanical tensor maps. Nature, (404):190-193, 2000. Macmillan Publishers Ltd. 235

7. H. Delinguette. Toward Realistic Soft-tissue Modeling in Medical Simulation. Proceedings of the IEEE, 86(3):512-523, March 1998. 235, 236

8. Pengcheng Shi, Albert J. Sinusas, R. Todd Constable, and James S. Duncan. Volumetric deformation using mechanics-based data fusion: Applications in cardiac motion recovery. International Journal of Computer Vision, November 1999. 235

9. J. Martin, A. Pentland, S. Sclaroff, and R. Kikinis. Characterization of Neuropathological Shape Deformations. IEEE Transactions on Pattern Analysis and Machine Intelligence, 20(2):97-112, February 1998. 236

10. Jean-Philippe Thirion and Guillaume Calmon. Deformation analysis to detect and quantify active lesions in 3d medical image sequences. Technical Report 3101, INRIA Sophia Antipolis, February 1997. 236

11. Stephen M. Pizer, Christina A. Burbeck, Daniel S. Fritch, Bryan S. Morse, Alan Liu, Shobha Murthy, and Derek T. Puff. Human Perception and Computer Image Analysis of Objects in Images. In DICTA-93 Digital Image Computing: Techniques and Applications, volume 1, pages 19-26, 1993. 236

12. G. Stetten and S. M. Pizer. Medial Node Models to identify and Measure Objects in Real-Time 3D Echocardiography. IEEE Transactions on Medical Imaging, 18(10):1025-1034, 1999. 236

13. S. M. Pizer, D. S. Fritsch, P. Yushkevich, V. Johnson, and E. Chaney. Segmentation, registration, and measurement of shape variation via image object shape. IEEE Transactions on Medical Imaging, pages 851-865, 1996. 236

14. L. D. Cohen and Cohen I. Finite Element Methods for Active Contour Models and Balloons for 2D and 3D Images. IEEE Transactions on Pattern Analysis and Machine Intelligence, 15:1131-1147, 1993. 236, 242

15. C. Davatzikos. Spatial Transformation and Registration of Brain Images Using Elastically Deformable Models. Computer Vision and Image Understanding, 66(2):207-222, May 1997. 236

16. P. Thompson and A. W. Toga. A Surface-Based Technique for Warping ThreeDimensional Images of the Brain. IEEE Transactions on Medical Imaging, 15(4):402-417, 1996. 236

17. R. Bajcsy and S. Kovacic. Multi-resolution Elastic Matching. Computer Vision, Graphics, and Image Processing, 46:1-21, 1989. 236

18. G. E. Christensen, S. C. Joshi, and M. I. Miller. Volumetric Transformation of Brain Anatomy. IEEE Transactions on Medical Imaging, 16(6):864-877, December 1997. 236

19. M. Bro-Nielsen and C. Gramkow. Fast Fluid Registration of Medical Images. In Visualization in Biomedical Computing (VBC'96), pages 267-276, 1996. 236

20. X. Papademetris, A. J. Sinusas, D. P. Dione, and J. S. Duncan. 3D Cardiac Deformation from Ultrasound Images. In MICCAI 1999: Medical Image Computing and Computer Assisted Intervention, pages 420-429. Springer, September 1999. 236

21. J. Park, D. Metaxas, and L. Axel. Analysis of left ventricular wall motion based on volumetric deformable models and MRI-SPAMM. Medical Image Analysis, 1(1):53-71, 1996. 236 
22. E. Haber, D. N. Metaxas, and L. Axel. Motion Analysis of the Right Ventricle from MR images. In MICCAI 1998: Medical Image Computing and Computer Assisted Intervention, pages 177-188. Springer, October 1998. 236

23. S. K. Kyriacou, C. Davatzikos, S. J. Zinreich, and R. N. Bryan. Nonlinear elastic registration of brain images with tumor pathology using a biomechanical model. IEEE Transactions on Medical Imaging, 18(7):580-592, july 1999. 236

24. O. C. Zienkewickz and R. L. Taylor. The Finite Element Method. McGraw Hill Book Co., 1987. 238, 239

25. Will Schroeder, Ken Martin, and Bill Lorensen. The Visualization Toolkit: An Object-Oriented Approach to 3D Graphics. Prentice Hall PTR, New Jersey, second edition, 1998. 239

26. B. Geiger. Three dimensional modeling of human organs and its application to diagnosis and surgical planning. Technical Report 2105, INRIA, 1993. 239

27. M. Kass, A. Witkin, and D. Terzopoulos. Snakes: Active Contour Models. International Journal of Computer Vision, 1(4):321-331, 1988. 242

28. O. Cuisenaire and B. Macq. Fast Euclidean Distance Transformation by Propagation Using Multiple Neighborhoods. Computer Vision and Image Understanding, 76(2):163-172, November 1999. 242

29. M. Ferrant, O. Cuisenaire, and B. Macq. Multi-Object Segmentation of Brain Structures in 3D MRI Using a Computerized Atlas. In SPIE Medical Imaging '99, volume 3661-2, pages 986-995, 1999. 243 